\title{
Interleukin-I $\beta$ inhibitors for the treatment of cryopyrin-associated periodic syndrome
}

\author{
This article was published in the following Dove Press journal: \\ The Application of Clinical Genetics \\ 25 January $201 \mathrm{I}$ \\ Number of times this article has been viewed
}

\section{Eugen Dhimolea}

Tufts University School of Medicine, Boston, MA, USA
Correspondence: Eugen Dhimolea Tufts University School of Medicine, 136 Harrison Avenue, Boston,

MA 02III, USA

Tel +I 6176360370

Fax +I 6176363676

Email eugen.dhimolea@gmail.com
Abstract: Cryopyrin-associated periodic syndrome (CAPS) comprises a group of rare, but severe, inherited autoinflammatory disorders associated with aberrant secretion of interleukin (IL)-1. These distinct conditions of autoinflammatory origin include Muckle-Wells syndrome, familial cold autoinflammatory syndrome, and neonatal-onset multisystem inflammatory disease (NOMID), which is also referred to as chronic infantile neurologic cutaneous and articular syndrome. Recently, this group of diseases has been associated with mutations in the NLRP3 gene that encodes for the protein cryopyrin, a component of the inflammasome complex that regulates the maturation and secretion of inflammatory cytokine IL-1 $\beta$. Immune cells from patients with NOMID secrete higher levels of active IL-1 $\beta$ compared with monocytes from healthy subjects. Overproduction of IL-1 is believed to promote aberrant inflammatory response in CAPS patients. Evidence supporting the clinical value of IL-1 $\beta$ in CAPS has been provided from the complete response of patients after treatment with IL-1 blocking agents.

Keywords: CAPS, IL-1 $\beta$, NLRP3, canakinumab, anakinra

\section{Introduction}

Cryopyrin-associated periodic syndrome (CAPS) is a spectrum of inherited autoinflammatory syndromes, which includes familial cold autoinflammatory syndrome (FCAS, formerly termed familial cold-induced urticaria), Muckle-Wells syndrome (MWS), and neonatal-onset multisystem inflammatory disease (NOMID, also called chronic infantile neurologic cutaneous and articular syndrome [CINCA]). These conditions share many clinical symptoms. Common features in CAPS patients include inflammation of the skin, eyes, bones, joints, and meninges, accompanied by severe fatigue, fever, myalgia, chronic anemia, and learning difficulties. Although these syndromes were initially considered to be distinct diseases, they are now thought to represent a continuum of related clinical subphenotypes. This review focuses on the recent advances in the treatment of these patients using anti-inflammatory biologic agents. In addition to improved treatment, the development of novel drugs has helped elucidate the role of the inflammation pathways in CAPS.

Interestingly, patients experiencing various forms of CAPS share a number of mutations of the NLRP3 (NOD-like receptor family, pyrin domain containing 3 ) gene. The NLRP3 gene is also known as CIAS1 (cold-induced autoinflammatory syndrome 1). NLRP3 encodes for the cryopyrin protein (NACHT, LRR, and PYD domains containing protein 3 [NALP3]), which is known to be involved in inflammation response and apoptosis. ${ }^{1}$ This observation has led to the hypothesis that the underlying pathological cause of CAPS may be of an autoinflammatory nature. However, a number 
of common anti-inflammatory drugs such as nonsteroidal agents, colchicine, immunosuppressants, and corticosteroids have been tested in CAPS patients with limited success. ${ }^{2}$ It has been reported that patients with NOMID have higher interleukin (IL)-1 $\beta$ serum levels compared with healthy individuals. ${ }^{3}$ IL- $1 \beta$ is a proinflammatory cytokine that is known to mediate the peripheral immune response during infection and inflammation. IL-1 $\beta$ is also involved in acute and chronic autoimmune diseases, diabetes, pain, and neurological disorders. ${ }^{4}$ Experimental data derived from animal model and in vitro studies suggest that IL- $1 \beta$ is a more potent mediator of inflammation than IL- $1 \alpha$ is. ${ }^{5}$ Targeting the IL- $1 \beta$ pathway has proved a successful approach in patients with CAPS. Among these newer therapies, anakinra (Kineret ${ }^{\circledR}$; Swedish Orphan Biovitrum, Stockholm, Sweden), rilonacept (Arcalyst $^{\circledR}$; Regeneron Pharmaceuticals, Tarrytown, NY, USA), and canakinumab (also known as ACZ885 and commercially as Ilaris ${ }^{\circledR}$; Novartis Pharma Stein AG, Stein, Swizerland) have shown remarkable efficacy and led to dramatic alleviations of CAPS symptoms.

\section{Review of the pathophysiology}

It has become evident in recent years that the innate immune system encompasses a highly complex signaling network, which is able to discriminate between the body's antigens and cells and external pathological elements. This function of the immune system is carried out by a large and diverse family of pathogen-recognition receptors (PRRs). One of the best known PRRs are toll-like receptors (TLRs), which are single, membrane-spanning, noncatalytic proteins that recognize structurally conserved molecules belonging to pathogenic micro-organisms. ${ }^{6}$ The microbes are recognized by TLRs after breaching physical barriers such as the skin or intestinal tract mucosa, which results in the activation of immune cell response. TLRs are found in a range of species, both vertebrates and invertebrates. The TLR gene sequence from various species, such as Caenorhabditis elegans, Drosophila, and mammals, shows a high degree of evolutionary conservation. ${ }^{7}$ Ten different members of the TLR family have been identified and characterized in humans. The receptors have been located on the plasma membrane and endosomal membrane of several cell types. Based on this evidence, it has been proposed that TLRs control both the extracellular space and the intracellular endosomal compartments.

The cytoplasmic domain of the receptor contains a toll/IL-1 receptor (TIR) region, which is a functional part of the signaling cascade downstream of IL-1 receptors. TLRs together with the IL-1 receptors belong to a receptor superfamily known as the interleukin-1 receptor/toll-like receptor superfamily. The members of this superfamily share the cytoplasmic TIR domain. The extracellular domain of TLRs is responsible for ligand binding and contains leucine-rich repeats (LRR) flanked by cystein-rich motifs. TLRs recognize and bind to specific molecular motifs borne by pathogens, called pathogen-associated molecular patterns (PAMPs). Ligands of TLRs include pathogenic antigens such as bacterial lipopolysaccharide (LPS), doublestranded viral RNA, and the bacterial protein flagellin. Binding of PAMPs induces conformational changes that lead to receptor dimerization, subsequent recruitment of TIR-domain-containing adapter proteins, and activation of downstream signaling pathways. ${ }^{8}$

The second largest gene family of PRRs is the NLR family, which was discovered in early $2000 .{ }^{9,10}$ NLR receptors are expressed in humans and mice and are homologous to so-called $\mathrm{R}$ (resistance) genes in plants, playing a role in protection against pathogens. ${ }^{11}$ The proteins encoded by these genes are located in the cytosol or on the plasma membrane. Similarly to TLRs, they function as detectors of the presence of pathogens and transducers of signals downstream of the MAP-kinase pathway. Activation of the NLR signaling cascade leads to cell death, which is considered an evolutionary primitive type of immune reaction in multicellular organisms. All NLR proteins have their cytoplasmic regions NACHT and LRR in common. The domain NACHT (domain present in neuronal apoptosis inhibitory protein [NAIP], CIITA, HET-E, and TP1) is located in the middle region of the molecule and is necessary for oligomerization. ${ }^{1}$ The domain LRRs (also known as NODs and CATERPILLERs) is found at the carboxy terminus and appears to be involved in a range of diverse processes required for host immune reactions against pathogens. ${ }^{12}$ In addition to these two regions there is a third, aminoterminal pyrin domain (PYD), which is shared by that of pyrin; it was first reported as a "putative pyrin-like protein" and is shared by many NLR family proteins.

Recently, it has been revealed that mutations in some genes belonging to the NLR family can cause abnormal secretion of inflammatory cytokines, which in turn may lead to the development of autoinflammatory diseases. ${ }^{2}$ This important finding has led to the reconsideration of the differential diagnosis and therapeutic approach in patients with recurrent episodes of fever and other symptoms of autoinflammation, malignancy, allergy, and other disorders of the immune system. The identification of genetic mutations in genes encoding for molecules that play a central role during the inflammatory response has centered attention on 
rare clinical conditions, which were consistently neglected in the past. The research in the field of NLRs has led to significant changes in the clinical management of patients with autoinflammatory conditions whose illnesses had not been correctly diagnosed for years. $^{2}$

\section{Genetic mutations and their role in CAPS}

Mutations linked with autoinflammatory diseases were first identified in the gene responsible for familial Mediterranean fever (FMF). The gene encodes for a protein initially called marenostrin, from the Latin mare nostrum, which refers to the Mediterranean Sea. However, the protein was renamed and is widely known as pyrin, referring to the Ancient Greek word for fever. ${ }^{13}$ Several other gene mutations associated with various autoinflammatory conditions have been identified since then. A study conducted in seven families affected by periodic fever syndromes revealed six different missense mutations of the $55 \mathrm{kDa}$ tumor necrosis factor receptor (TNFR1), five of which disrupt evolutionary conserved extracellular disulfide bonds. ${ }^{14}$ Analysis of cells from four patients with periodic fever syndromes revealed reduced activities of mevalonate kinase (encoded by the gene MVK), an enzyme involved in isoprenoid biosynthesis. DNA sequence analysis in these patients revealed several mutations that caused reduced enzymatic activity. ${ }^{15,16}$

Another study conducted in patients with autoinflammatory diseases revealed four distinct mutations in the NLRP3 gene (also known as CIAS1) that statistically correlated with the disorder in three families with FCAS and one family with MWS. ${ }^{17}$ NLRP3 is known to play a key role in the regulation of inflammatory response. Since the discovery of these first NLRP3 mutations, additional mutations of the same gene have been reported in FCAS, Muckle-Wells syndrome, and CINCA (also known as NOMID). ${ }^{18,19}$ Three missense mutations were also identified in the nucleotidebinding domain (NBD) of CARD15/NOD2 in four French and German families with Blau syndrome. ${ }^{20}$

The identification of NLRP3 gene mutations has led to the recognition of FCAS, MWS, and CINCA as a continuum of pathologically related conditions called cryopyrin. Several NLRP3 missense mutations that have been described in CAPS patients support a genetic etiology for this disease. The majority of these mutations are found in the NACHT domain of the cryopyrin protein. The degree of protein conformational changes and subsequent effect on the enzymatic function depends on the exact location of the mutation and usually leads to synthesis of constitutively activated protein.
The impact of the mutation on protein functionality may affect the severity of the symptoms. FCAS, for instance, is the mildest form of CAPS and is characterized by fever, cold-induced urticarial rash, and arthralgia. CINCA includes neonatal-onset high fever, mental retardation, aseptic meningitis, arthritis with bone overgrowth, sensory deafness, papilledema, and secondary amyloidosis leading to mortality, and is generally considered the most severe form of CAPS. The symptoms of MWS are of an intermediate phenotype. Cases that overlap between FCAS and MWS or MWS and CINCA have been reported as well.

The inflammasome is a multiprotein complex consisting primarily of caspase-1, apoptosis-associated speck-like protein containing a CARD (ASC), and the NALP protein. The recruitment of the components that constitute this complex depends on the presence of activators that initiate inflammasome assembly. The inflammasome is responsible for activation of inflammatory processes and has been shown to induce cell pyroptosis through activation of caspase-1. The activation of caspase-1 induces the enzymatic processing of several precursors of inflammatory cytokines such as pro-IL-1 $\beta$ and pro-IL-18 into their mature forms, in which they are subsequently secreted by the cells. ${ }^{21}$ It was quickly recognized that the structure and functions of the inflammasome resemble those of the apoptosome assemble. Apoptosome is a heptameric complex consisting of apoptosis protease activating factor-1 (Apaf-1), which is induced by the release of cytochrome $\mathrm{C}$ from mitochondria. Apoptosome assembling involves the recruitment of precursor protein procaspase- 9 and its modification into mature and active caspase-9. It has been found that Apaf has high structural similarity with NLR family proteins, which are thought to trigger or regulate host defense mechanisms through formation of signaling platforms that subsequently lead to activation of inflammatory caspases and NF-kB. ${ }^{22}$ It is believed that the mechanism of apoptosome formation by Apaf-1 could provide insights into the activation mechanisms of several NLR proteins, including NLRP1, NLRP3, and NLRC4, for the construction of the inflammasome. The study of inflammasome complex assembly has revealed that the recruitment of NLRP1 and ASC is necessary for the activation of caspase- 1 and pro-IL-1 maturation into IL-1. ${ }^{23}$ According to this proposed model of inflammasome activation, caspase- 5 binds to the CARD domain of NLRP1 while the PYD region of caspase1-bound ASC protein interacts with the respective PYD domain of NLRP1. This model is corroborated by the clinical studies reporting that mutations in the NLRP3 gene are frequently found in patients 
with FCAS and MWS. ${ }^{17,24}$ Furthermore, the hypothesis of NLRP3 mutation-induced aberrant regulation of IL-1 secretion is supported by ex vivo experiments showing that abnormally high levels of the cytokine were produced by monocytes isolated from an MWS patient carrying the R260W mutation. Overproduction of IL-1 $\beta$ has also been reported in monocytes carrying other NLRP3 mutations. ${ }^{25}$

\section{Advances in CAPS therapeutics}

Blocking the IL-1 signaling pathway has become an important strategy in the pharmaceutical treatment of CAPS patients (Table 1). Anakinra is a recombinant human IL-1 receptor antagonist that has been approved for the treatment of rheumatoid arthritis in 20 countries. In 2003, anakinra was reported to control inflammatory symptoms in CAPS patients within hours after the first injection. ${ }^{26}$ Although this group reported data for only two patients with MWS, this was the first clinical proof of concept for IL-1-targeting drugs in CAPS. This first report was soon followed by a retrospective review of medical records of 22 individuals from 13 families with autoinflammatory diseases associated with CIAS-1/NALP3 mutations. Fifteen of these patients, most of whom had MWS, received anakinra for a 39-month period. The data analysis showed that all the patients who received anakinra achieved serologic remission and complete resolution of fever, rash, conjunctivitis, and rheumatic symptoms without any adverse effects. Anakinra was also able to prevent cold-associated acute inflammation in patients with FCAS during an experimental cold challenge protocol to study the acute inflammatory mechanisms occurring after a general cold exposure in FCAS patients. ${ }^{27}$ In addition, 18 patients with NOMID (12 with identifiable CIAS1 mutations) who received anakinra had a rapid response with the disappearance of rash and improvement of inflammation biomarkers profile. ${ }^{28}$ However, anakinra had limited effect in a case of severe CINCA syndrome with a novel G307V cryopyrin mutation. ${ }^{29}$ In some patients with MWS, treatment with anakinra led to recovery from deafness. ${ }^{30}$ Anakinra is usually administered daily by subcutaneous injection, which increases the difficulty in the management of patients with CAPS. IL-1 blocking agents with longer half-lives have now been developed and tested in various conditions.

Rilonacept is a fusion protein encompassing the Fc portion, an IgG1 molecule, and the extracellular domain of the IL-1 receptor. Rilonacept inhibits IL-1 action by binding and blocking circulating cytokine molecules (IL-1 $\alpha$ and IL-1 $\beta$ ) with high affinity. Rilonacept belongs to a class of biologics called "cytokine traps". The safety and efficacy of $300 \mathrm{mg}$ rilonacept administered by subcutaneous injection was tested in an open-label trial involving five patients with FCAS in an open-label pilot study to evaluate the safety and efficacy of rilonacept. ${ }^{31}$ All patients remained off treatment until a clinical flare occurred. After flare recurrence, patients were again treated with $300 \mathrm{mg}$ rilonacept and then given maintenance doses of $100 \mathrm{mg}$ weekly. In cases where FCAS was not completely controlled after the follow-up treatment, the patients received dosage increases of $160 \mathrm{mg} /$ week to $320 \mathrm{mg} /$ week during an intrapatient dosage escalation phase. In all patients, clinical symptoms typically induced by cold (rash, fever, and joint pain/swelling) improved within 6 to 10 days of rilonacept administration, and biomarkers of inflammation (erythrocyte sedimentation rate, high-sensitivity C-reactive protein [CRP], and serum amyloid A [SAA]) showed statistically significant reductions at the maintenance dose of $100 \mathrm{mg}$. In general, dosage escalation resulted in improvement of symptoms, including rash and joint pain. The drug was well tolerated and no serious adverse events were seen in a 2-year period.

A second clinical study to assess the efficacy and safety of rilonacept involved 47 adult patients with CAPS (44 with FCAS and three with MWS; CINCA patients were not included), as defined by mutations in the causative NLRP3 (CIAS1) gene and pathognomonic symptoms. The study was separated into two consecutive Phase III studies. ${ }^{32}$ Disease activity and symptom improvement was evaluated using a validated self-administered questionnaire in which patients rated the severity of their main symptoms (rash, feeling of fever/chills, joint pain, eye redness/pain, and fatigue). The first study consisted of a 6-week, randomized, double-blind comparison of weekly subcutaneous injections of rilonacept (160 mg) versus placebo. In this arm of the trial, rilonacept therapy lowered the group mean composite symptom score by $84 \%$, compared with $13 \%$ with placebo therapy (primary endpoint; $P<0.0001$ versus placebo). Rilonacept also significantly improved all other efficacy endpoints in the first part of the study (including numbers of multisymptom and single-symptom disease flare days, single-symptom scores, physicians' and patients' global assessments of disease activity, limitations in daily activities, and CRP and SAA levels). The second part of the study involved 9 weeks of single-blind treatment with rilonacept (part A), followed by a 9-week, randomized, double-blind, placebo-controlled withdrawal procedure (part B). Similarly to the first study, primary efficacy was evaluated using a validated composite key symptom score. In study 2, rilonacept was superior to placebo for maintaining the improvements, as shown by all efficacy parameters (primary endpoint; $P<0.0001$ versus 
placebo). Rilonacept was generally well tolerated; the most common adverse events were injection site reactions.

Canakinumab is a human anti-IL-1 $\beta$ monoclonal antibody. In June 2009 the US Food and Drug Administration (FDA) approved the drug for the treatment of FCAS and MWS. Its mechanism of action is based on the cytokine binding and consequent neutralization of IL-1 $\beta$ (but not IL-1 $\alpha$ ) signal, resulting in suppression of inflammation in patients with disorders of autoinflammatory origin. Canakinumab was produced using the UltiMab ${ }^{\mathrm{TM}}$ technology developed by Medarex, Princeton, NJ, USA. The efficacy and safety of canakinumab were initially assessed in a proof of concept study for patients with MWS carrying the NLRP3 gene mutation. ${ }^{33}$ The drug was administered intravenously at a dose of $10 \mathrm{mg} / \mathrm{kg}$. Patients who relapsed received a second intravenous infusion of $1 \mathrm{mg} / \mathrm{kg}$. A rapid improvement in MWS symptoms was reported within 2 days and complete remission after 8 days. Remission had a median duration of 185 days, and additional dosing after relapse resulted in a median symptoms-free period of 90 days.

A single-center interim open-label Phase II study evaluated the safety and efficacy of canakinumab. ${ }^{34}$ All patients (12 in total: seven females and five males) participating in this study carried the NLRP3 mutation and had confirmed active disease requiring medical intervention. Patients received a single subcutaneous injection of canakinumab (150 mg for adults; $2 \mathrm{mg} / \mathrm{kg}$ for children). Patients who relapsed or had incomplete response within 7 days received additional injections of $5 \mathrm{mg} / \mathrm{kg}$ canakinumab via intravenous application. Complete response was defined by i) physician's global assessment of disease activity (PHY) and assessment of skin disease (SKD) as $\leq 2$ on a 5 -point scale ( $1=$ absent, $2=$ minimal, $3=$ mild, $4=$ moderate, $5=$ severe), and ii) normal serum values $(<10 \mathrm{mg} / \mathrm{L})$ of CRP and/or SAA. Patient median age varied between 4.3 and 47.4 years (median 27.6 years, including four children below 14 years). Nine of the patients had been under anakinra regimen up to $8 \mathrm{mg} / \mathrm{kg}$ prior to the canakinumab trial. According to study analysis, all 12 patients treated with canakinumab achieved a complete and rapid clinical and serological response. Two children and one adult were subjected to the adjusted intravenous canakinumab doses. Median time to relapse and redosing was 92 days (nine patients) after the first treatment cycle and 66 days (six patients) after the second treatment cycle. Two patients remained relapse free for 106 days. Canakinumab was generally well tolerated, and the major adverse effects were upper respiratory tract infections (seven events) and elevated pancreas amylase and lipase (two events), and one patient experienced a vertigo episode.

Another important canakinumab study in patients with CAPS consisted of a three-part, double-blind, placebocontrolled, randomized withdrawal trial of 48-week total duration. ${ }^{35}$ Thirty-five patients (9-74 years of age) participated in part 1 , receiving $150 \mathrm{mg}$ of canakinumab subcutaneously for 8 weeks. Patients who had complete response to treatment entered part 2 and received either $150 \mathrm{mg}$ of canakinumab or placebo every 8 weeks for up to 24 weeks. The patients who completed part 2 or relapsed proceeded to part 3, which consisted of at least two more doses of canakinumab for a 16-week, open-label, active treatment period. The therapeutic responses were evaluated using disease activity scores and analysis of CRP and SAA levels. A complete response was defined as i) patient ratings of "minimal" or "better" for PHY and SKD, and ii) serum levels of CRP and SAA less than $10 \mathrm{mg} / \mathrm{L}$. A disease flare (relapse) was defined as a CRP and/or SAA values greater than $30 \mathrm{mg} / \mathrm{L}$ and either a score of "mild" or "worse" for PHY or a score of "minimal" or "worse" for PHY and SKD.

In part 1 of the study, 34 of the 35 patients (97\%) showed a complete response to canakinumab. Markers of inflammation CRP and SAA returned to normal levels within 8 days of treatment in most patients. Normalization in the mean CRP and SAA values was sustained throughout part 1 in patients continuously treated with canakinumab. Patients with complete responses and without disease flare from part 1 were randomized $(1: 1)$ to canakinumab or placebo in part 2 . From the 31 patients who entered part 2, all 15 patients receiving canakinumab remained in remission, but relapse occurred in

Table I Interleukin-I $\beta$ inhibitors used in the treatment of cryopyrin-associated periodic syndrome

\begin{tabular}{|c|c|c|c|}
\hline \multirow[t]{2}{*}{ Type } & Anakinra & Rilonacept & Canakinumab \\
\hline & IL-IR recombinant protein & IL-IR-Fc dimeric fusion & Monoclonal antibody \\
\hline Trade name & Kineret $^{\circledR}$ & Arcalyst $^{\circledR}$ & Ilaris $^{\circledR}$ \\
\hline Mechanism of action & Interleukin-I blocker & Interleukin-I trap & Interleukin-I $\beta$ blocker \\
\hline Dosage & $\mathrm{I}-8 \mathrm{mg} / \mathrm{kg}$ & $100-300 \mathrm{mg}$ & $\mathrm{I}-10 \mathrm{mg} / \mathrm{kg}$ \\
\hline Administration & Daily & Weekly & Bimonthly \\
\hline Status & Under development & Approved & Approved \\
\hline
\end{tabular}


13 of the 16 patients $(81 \%)$ receiving placebo $(P<0.001)$. At the end of part 2, median CRP and SAA values were normal $(<10 \mathrm{mg}$ per liter for both measures) in patients receiving canakinumab but increased in those receiving placebo $(P<0.001$ and $P=0.002$, respectively). All patients treated with canakinumab in part 2 had absent or minimal symptoms. Of the 31 patients, 28 (90\%) completed the third part of the study in remission. Reintroduction of canakinumab in part 3 resulted in normalization of CRP and SAA values. The incidence of suspected infections in part 2 was greater in the canakinumab group than in the placebo group $(P=0.03)$, suggestive of canakinumab immunosupressing activity. ${ }^{35}$ Additional trials are currently ongoing to assess the efficacy and safety of canakinumab in CAPS patients. ${ }^{36}$

\section{Conclusions}

Targeting cytokines involved in inflammatory processes has become a common approach in the field of new therapeutics for autoinflammatory diseases. Cryopyrin-associated periodic syndromes are linked to mutations occurring on the gene encoding the NLR family protein NLRP3. The constitutively activated protein leads to cleavage of pro-IL-1, resulting in oversecretion of mature IL-1 and promotion of inflammation. Therefore, dysregulated IL- $1 \beta$ activity has become the target of choice for the treatment of CAPS patients. Several IL-1 blocking agents are being developed for a range of therapeutic indications. Anakinra was the first IL-1 blocker to have shown efficacy in multiple autoimmune disorders and inflammatory conditions linked to NLRP3 mutations (MWS and NOMID). However, rilonacept (a product of Regeneron Pharmaceuticals) was the first IL-1 blocker to be approved by the FDA for the treatment of CAPS disorders. Because of its ability to block both IL-1 isoforms, it has been suggested that rilonacept might have better inhibitory effect in vivo compared with other IL-1 blockers.

Canakinumab is the second IL-1 blocker that has been approved for the treatment of CAPS and is currently undergoing trials for autoimmune disorders. Unlike anakinra and rilonacept, which block the signals of both the IL- $1 \alpha$ and IL- $1 \beta$ isoforms, canakinumab is a highly specific IL- $1 \beta$ blocker and does not interfere with other IL-1-activated pathways. Due to its greater half-life, canakinumab can block IL-1 $\beta$ action for a longer period of time without the need for frequent injections or high doses. Notably, in patients with CAPS, canakinumab is administered subcutaneously every 2 months, as opposed to the weekly and daily injections with rilonacept and anakinra, respectively.
Diagnosis of patients with CAPS has been made mainly based on the characteristics of the inflammatory symptoms of individual patients. The discovery of gene mutations associated with abnormal inflammatory response has allowed for the introduction of clinical genetic tests, resulting in more accurate diagnosis. The results from the ongoing clinical trials are expected to advance our knowledge regarding the clinical value of IL-1 blockers in CAPS patients and help us to understand the biological relevance of IL-1 $\beta$ in autoinflammatory disorders.

\section{Disclosure}

No conflicts of interest were declared in relation to this paper.

\section{References}

1. Damiano JS, Oliveira V, Welsh K, et al. Heterotypic interactions among NACHT domains: implications for regulation of innate immune responses. Biochem J. 2004;381(Pt 1):213-219.

2. Kubota T, Koike R. Cryopyrin-associated periodic syndromes: background and therapeutics. Mod Rheumatol. 2010;20:213-221.

3. Neven B, Prieur AM, Quartier dit Maire P. Cryopyrinopathies: update on pathogenesis and treatment. Nat Clin Pract Rheumatol. 2008;4:481-489.

4. Dinarello CA. Therapeutic strategies to reduce IL-1 activity in treating local and systemic inflammation. Curr Opin Pharmacol. 2004;4: 378-385.

5. Pascual V, Allantaz F, Arce E, et al. Role of interleukin-1 (IL-1) in the pathogenesis of systemic onset juvenile idiopathic arthritis and clinical response to IL-1 blockade. J Exp Med. 2005;201:1479-1486.

6. Hansson GK, Edfeldt K. Toll to be paid at the gateway to the vessel wall. Arterioscler Thromb Vasc Biol. 2005;25:1085-1087.

7. Roach JC, Glusman G, Rowen L, et al. The evolution of vertebrate Toll-like receptors. Proc Natl Acad Sci U S A. 2005;102:9577-9582.

8. Arancibia SA, Beltrán CJ, Aguirre IM, et al. Toll-like receptors are key participants in innate immune responses. Biol Res. 2007;40:97-112.

9. Inohara N, Nuñez G. The NOD: a signaling module that regulates apoptosis and host defense against pathogens. Oncogene. 2001;20: 6473-6481.

10. Harton JA, Linhoff MW, Zhang J, et al. Cutting edge: CATERPILLER: a large family of mammalian genes containing CARD, pyrin, nucleotide-binding, and leucine-rich repeat domains. J Immunol. 2002; 169:4088-4093.

11. Jones JD, Dangl JL. The plant immune system. Nature. 2006;444: 323-329.

12. Inohara N, Chamaillard, McDonald C, et al. NOD-LRR proteins: role in host-microbial interactions and inflammatory disease. Annu Rev Biochem. 2005;74:355-383.

13. [No authors listed] Ancient missense mutations in a new member of the RoRet gene family are likely to cause familial Mediterranean fever. The International FMF Consortium. Cell. 1997;90:797-807.

14. McDermott MF, Aksentijevich I, Galon J, et al. Germline mutations in the extracellular domains of the $55 \mathrm{kDa}$ TNF receptor, TNFR1, define a family of dominantly inherited autoinflammatory syndromes. Cell. 1999;97:133-144.

15. Houten SM, Kuis W, Duran M, et al. Mutations in MVK, encoding mevalonate kinase, cause hyperimmunoglobulinaemia $\mathrm{D}$ and periodic fever syndrome. Nat Genet. 1999;22:175-177.

16. Drenth JP, Cuisset L, Grateau G, et al. Mutations in the gene encoding mevalonate kinase cause hyper-IgD and periodic fever syndrome. International Hyper-IgD Study Group. Nat Genet. 1999;22:178-181. 
17. Hoffman HM, Mueller JL, Broide DH, et al. Mutation of a new gene encoding a putative pyrin-like protein causes familial cold autoinflammatory syndrome and Muckle-Wells syndrome. Nat Genet. 2001; 29:301-305.

18. Aksentijevich I, Nowak M, Mallah M, et al. De novo CIAS1 mutations, cytokine activation, and evidence for genetic heterogeneity in patients with neonatal-onset multisystem inflammatory disease (NOMID): a new member of the expanding family of pyrin-associated autoinflammatory diseases. Arthritis Rheum. 2002;46:3340-3348.

19. Feldmann J, Prieur AM, Quartier P, et al. Chronic infantile neurological cutaneous and articular syndrome is caused by mutations in CIAS1, a gene highly expressed in polymorphonuclear cells and chondrocytes. Am J Hum Genet. 2002;71:198-203.

20. Miceli-Richard C, Lesage S, Rybojad M, et al. CARD15 mutations in Blau syndrome. Nat Genet. 2001;29:19-20.

21. Mariathasan S, Newton K, Monack DM, et al. Differential activation of the inflammasome by caspase-1 adaptors ASC and Ipaf. Nature. 2004; 430:213-218.

22. Proell M, Riedl SJ, Fritz JH, et al. The Nod-like receptor (NLR) family: a tale of similarities and differences. PLoS One. 2008;3:e2119.

23. Martinon F, Burns K, Tschopp J. The inflammasome: a molecular platform triggering activation of inflammatory caspases and processing of proIL-beta. Mol Cell. 2002;10:417-426.

24. Aganna E, Martinon F, Hawkins PN, et al. Association of mutations in the NALP3/CIAS1/PYPAF1 gene with a broad phenotype including recurrent fever, cold sensitivity, sensorineural deafness, and AA amyloidosis. Arthritis Rheum. 2002;46:2445-2452.

25. Dowds TA, Masumoto J, Zhu L, et al. Cryopyrin-induced interleukin 1 beta secretion in monocytic cells: enhanced activity of disease-associated mutants and requirement for ASC. J Biol Chem. 2004;279:21924-21928.

26. Hawkins PN, Lachmann HJ, McDermott MF. Interleukin1 -receptor antagonist in the Muckle-Wells syndrome. N Engl J Med. 2003;348:2583-2584.
27. Hoffman HM, Rosengren S, Boyle DL, et al. Prevention of coldassociated acute inflammation in familial cold autoinflammatory syndrome by interleukin-1 receptor antagonist. Lancet. 2004;364: 1779-1785.

28. Goldbach-Mansky R, Dailey NJ, Canna SW, et al. Neonatal-onset multisystem inflammatory disease responsive to interleukin-1beta inhibition. N Engl J Med. 2006;355:581-592.

29. Matsubara T, Hasegawa M, Shiraishi M, et al. A severe case of chronic infantile neurologic, cutaneous, articular syndrome treated with biologic agents. Arthritis Rheum. 2006;54:2314-2320.

30. Mirault T, Launay D, Cuisset L, et al. Recovery from deafness in a patient with Muckle-Wells syndrome treated with anakinra. Arthritis Rheum. 2006;54:1697-1700.

31. Goldbach-Mansky R, Shroff SD, Wilson M, et al. A pilot study to evaluate the safety and efficacy of the long-acting interleukin-1 inhibitor rilonacept (interleukin-1 Trap) in patients with familial cold autoinflammatory syndrome. Arthritis Rheum. 200;58: 2432-2442.

32. Hoffman HM, Throne ML, Amar NJ, et al. Efficacy and safety of rilonacept (interleukin-1 Trap) in patients with cryopyrin-associated periodic syndromes: results from two sequential placebo-controlled studies. Arthritis Rheum. 2008;58:2443-2452.

33. Lachmann HJ, Jung T, Bobadilla M, et al. Treatment of Muckle Wells syndrome with a fully human anti-IL-1beta monoclonal antibody (ACZ885) - initial results from a proof of concept study. Ann Rheum Dis. 2006;65:76.

34. Kuemmerle-Deschner JB, Tzaribachev N, Hansmann S, et al. Long lasting response to ACZ885 in patients with Muckle-Wells syndrome (MWS). Ann Rheum Dis. 2008;67:104.

35. Lachmann HJ, Kone-Paut I, Kuemmerle-Deschner JB, et al. Canakinumab in CAPS Study Group. Use of canakinumab in the cryopyrin-associated periodic syndrome. $N$ Engl J Med. 2009;360:2416-2425.

36. Dhimolea E. Canakinumab. $m A b s$. 2010;2:3-13.
The Application of Clinical Genetics

\section{Publish your work in this journal}

The Application of Clinical Genetics is an international, peer-reviewed open access journal that welcomes laboratory and clinical findings in the field of human genetics. Specific topics include: Population genetics; Functional genetics; Natural history of genetic disease; Managemen of genetic disease; Mechanisms of genetic disease; Counselling and

\section{Dovepress}

ethical issues; Animal models; Pharmacogenetics; Prenatal diagnosis; Dysmorphology. The manuscript management system is completely online and includes a very quick and fair peer-review system, which is all easy to use. Visit http://www.dovepress.com/testimonials.php to read real quotes from published authors. 